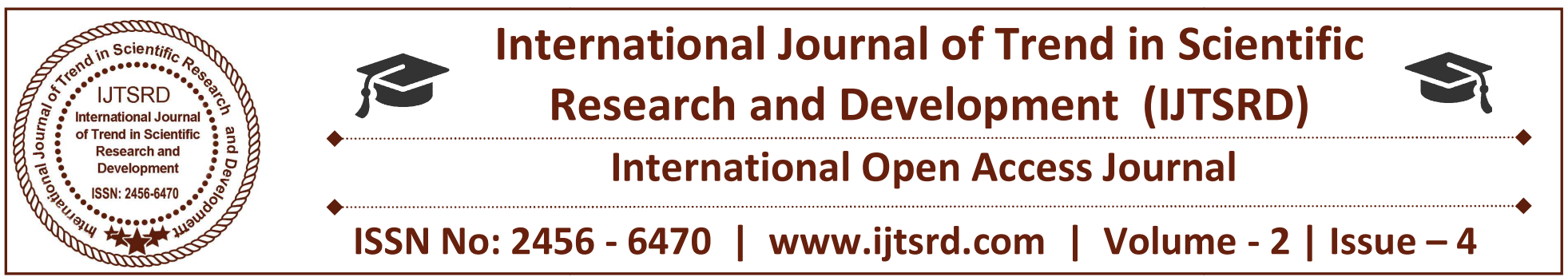

\title{
A Study on Sixth Sense Technology
}

\author{
Mrs. Ashwini $\mathbf{N}^{1}$, Ashwini $\mathbf{K}^{2}$ \\ ${ }^{1}$ Assistant Professor, ${ }^{2}$ Student \\ Department of ISE, BMSIT\&M, Bengaluru, Karnataka, India
}

\begin{abstract}
This paper discusses regarding 'Sixth Sense technology' that may be a wearable gestural interface that augments the physical world around United States of America with digital data and lets United States of America use natural hand gestures to act thereupon data.
\end{abstract}

Keywords: component, formatting, style, styling, insert

\section{INTRODUCTION}

Sixth Sense in scientific terms is outlined as further Sensory Perception or in brief E.S.P. It involves the reception of data not gained through any of the 5 senses. Neither is it taken from any experiences from the past or notable. Intuition aims to additional seamlessly integrate on-line info and school into standard of living. By creating out there info required for decision-making on the far side what we've got access to with our 5 senses, it effectively provides users an intuition. Info is hold on historically on a paper or a digital device. Intuition technology bridges this gap between tangible and intangible world of digital info and permitting U.S.A. to act with this via natural hand gestures [1]. The ultimate power of intuition lies at intervals the potential it holds to attach web with the $\$ 64000$ world and superimposing the information on the planet itself. though forthcoming technologies like five pen laptop technology enable U.S.A. to hold computers aboard with U.S.A. in our pockets, a link between the digital devices we will carry and its interactions with planet, additionally our speech, has not however been found. The accomplishment here is that intuition identifies the objects around oneself, allow us to access the info the knowledge the data $\}$ within the manner we wish and displays that information likewise, all this within the most simplest of the ways in which. Felicitous awareness of this technology can purpose to even more development and use of this technology, that inturn can aid in getting info and in operation any kind of perform much at any time [2].It has the potential to fully modification man's perspective concerning real objects and there'll be a skinny and slender line between our planet and also the virtual world. It'll create our world simply accessible by introducing the digital world into it [3]. Intuition Technology acknowledges the objects around U.S.A. and makes U.S.A. act with the data in any manner we'd like while not the employment of electronic gadgets mistreatment straightforward hand gestures. It may be used as fifth sense for unfit individuals. People will create their own applications consistent with their would like. Intuition technology clearly became a clear program so creating life easier [4].Hidden Markova models, time delay neural networks, multi-layer perceptron and model matching are wide used for gesture computing technology. Mathematical logic has not been wide employed in the event of gesture computing systems. This approach contains a large quantity of potential to bring a forceful modification in digital world by creating it additional human. The improvement may be achieved with the assistance of higher hardware specifications [5].

\section{Design Method}

The sixth sense device comprises of

1. Wearable projector

2. Mobile device

3. Speech IC

4. Mirror 


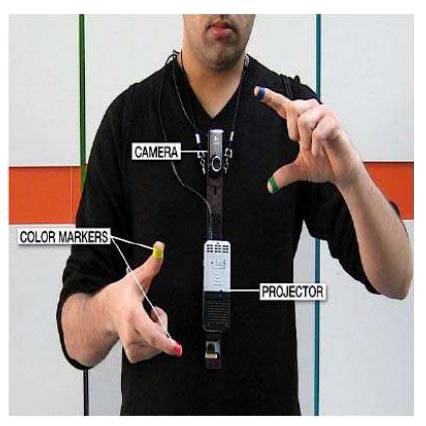

Fig 1. Components of Sixth Sense

The insight device may be a mini projector in addition to a speech IC and a cellular telephone, that acts as a pc and our association to the cloud, all the data keep on the online. The elements are controlled by or communicated with a mobile computing machine carried within the user's pocket. The hardware components are coupled in an extremely pendant like mobile wearable device every the speech IC and additionally the projector are connected to the mobile computer at intervals the user's pocket. The projector, comes the visual knowledge enabling surfaces, walls and physical objects around the user to be used as interfaces. Whereas the speech IC stores commands that were trained by the user and executes the corresponding action through the projector, enabling the actions from the mobile device. A faraway computer can also be connected that gathers info from user, processes it, searches the web for relevant execution of the command and returns the cause real time to the user. The speech IC is trained with often used operational info and so it acts as AN info for storing all such commands. There evolved many speech integrated circuits with fabulous technical aspects to be embedded for giant moderately applications. There are three ways in which for speech recognition and language understanding. 1. Serviceable processors meant for embedded applications. 2. Bespoken integrated circuits for speech recognition and language undererstanding.3.implementing speech recognition and language understanding as an area of larger electric circuit at intervals the device. Some integrated circuits is employed for fewer than fifteen words that have a menu primarily based variety of interaction whereas various ASIC integrated circuits is employed for several words that possess communication understanding. The IC are trained with a sophisticated neural network to recognise the commands and activate it correspondingly.

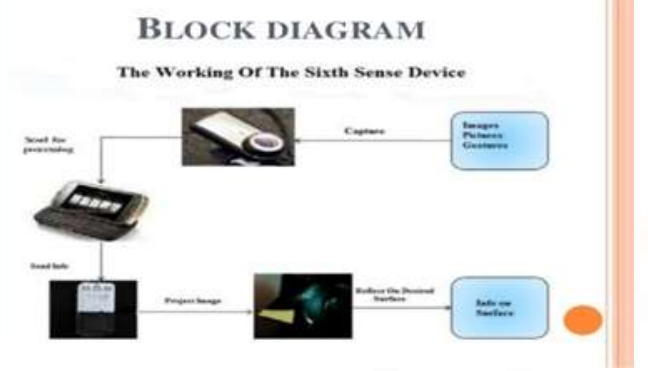

Fig 2. Block Diagram

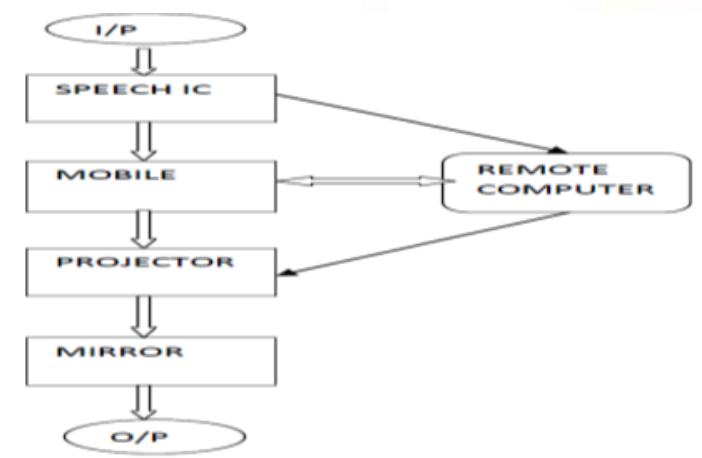

The speech IC is at the start trained with the words or commands. The user offers the input as commands and once such analog speech is received to the IC, the data is born-again into digital and is shipped to the mobile device the mobile device activates the command and is given successively to the projector. The projector output is seen on the screen through the mirror for correct projection from the projector that is wearable within the body. For additional advanced operations and for accessing internet that is our future work, may be accessed from the remote laptop at the same time and projected as before. 
Analysis of Both Methods

\begin{tabular}{|c|c|c|}
\hline & DIRECT MANIPULATION & NATURAL LANGUAGE \\
\hline STRENGTHS & $\begin{array}{l}\text { 1. Intuitive } \\
\text { 2. Consistent look feel } \\
\text { 1. 3.Options apparent } \\
\text { 3. Fail safe } \\
\text { 2. Direct engagement with the } \\
\text { object }\end{array}$ & $\begin{array}{ll}\text { 1. } & \text { Intuitive } \\
\text { 2. } & \text { Description } \\
\text { 3. } & \text { Context } \\
\text { 1. } & \text { 4.Asynchronous }\end{array}$ \\
\hline WEAKNESSES & $\begin{array}{l}\text { 1. Description } \\
\text { 2. Anaphora } \\
\text { 3. Operation on sets } \\
\text { 2. 4. Delayed actions difficult }\end{array}$ & $\begin{array}{l}\text { 1. Coverage's opaque } \\
\text { 2. Overkill for short and frequent queries } \\
\text { 3. Difficulty of establishing and navigating } \\
\text { context } \\
\text { 4. Error prone } \\
\text { 5. 5. Anaphora problematic }\end{array}$ \\
\hline
\end{tabular}

\section{Applications}

\section{A. Calculator}

You can use the intuition to project an input device onto your hand as shown in Fig. 3, then we are able to use that virtual input device to calculate values. Not solely this, it is created to project keyboard furthermore, thus eliminating use of physical keyboard.

Fig. 3. Make a calculator

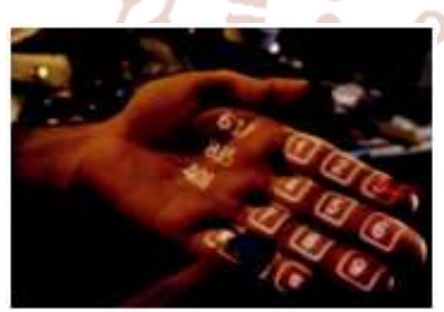

\section{B. Check Time}

Check time, by drawing circle on radio carpal joint, then projector can project watch on radio carpal joint as shown in Fig. 4. Thence there's no a lot of have to be compelled to wear watches obtainable, and additionally not wasting cash on branded watches.

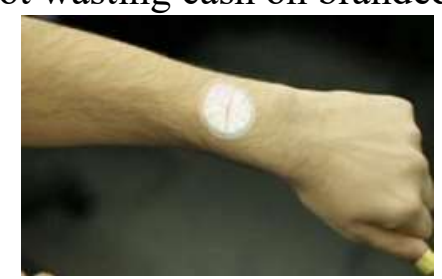

Fig 4. Check time

\section{Drawing Application}

Draw an image on any desired surface simply by moving the forefinger within the air as seen in Fig one.4.3. Hence, mistreatment this technology we will do drawings while not use of pencil or color and paper. And even be ready to save our drawings.

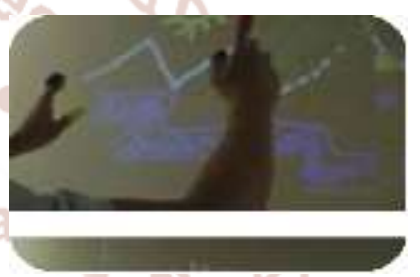

Fig 5. Draw Applications

D. Viewing Maps

We can read maps and additionally navigate. We will additionally zoom into the map simply by creating gestures like pinch in and out as seen in Fig. 5. Therefore there'll be no ought to carry large paper maps at the side of United States once we arrange for a visit.

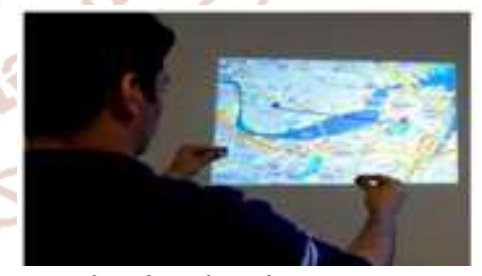

Fig 6. Viewing Maps

\section{E. Zooming Features}

We can focus and out by hand gestures by pinching fingers of each hands to focus and build each hands nearer to every alternative to zoom even as shown in Fig. 6.

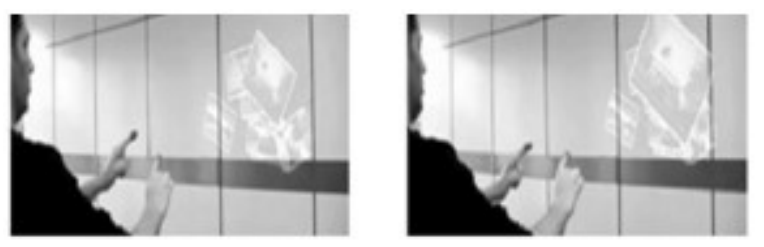

Fig. 7. Zoom in and out 


\section{F. Get Product Information}

Get info concerning product in our hand and Amazon offers rating to create a right call to shop for simply by showing the merchandise ahead of camera, the camera capture its image then in process it's for the knowledge from the Amazon web site. Therefore, we tend to don't have to open our web browsers on every occasion to appear for the knowledge.

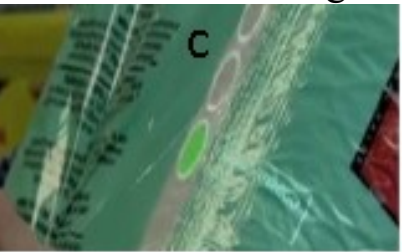

Fig 8. Product Information

\section{G. Get Book Information}

Get the data of the book and it's rating from the Amazon book store or the other web site. This device would prove a blessing to the book lovers simply by showing the duvet page of the book to the camera, the camera captures its image and whereas process it searches the rating for the book from.

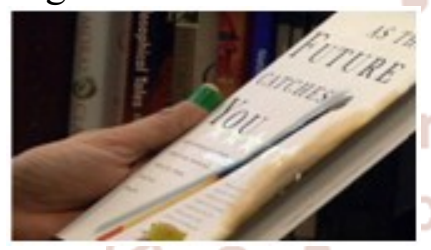

Fig 9. Book Rating

\section{H. Take Pictures}

Take an image simply by creating photograph frame or parallelogram by fingers. The photograph are going to be taken and it'll be placed in good phone's memory. An individual will size and appearance for the photograph whenever required by protruding it to be had or any surface.

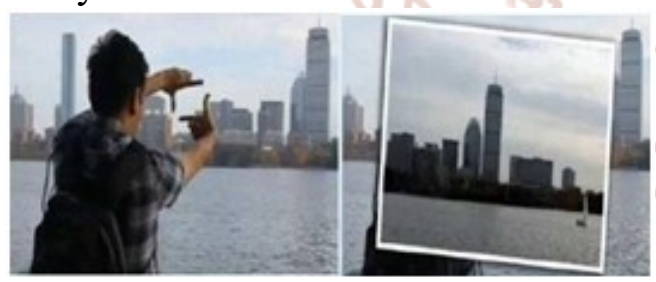

Fig 10. Taking Pictures

\section{Viewing weather information}

We can conjointly read this day's weather info simply by showing map on paper or any surface to the camera. The weather info is searched from the web by the good phone.

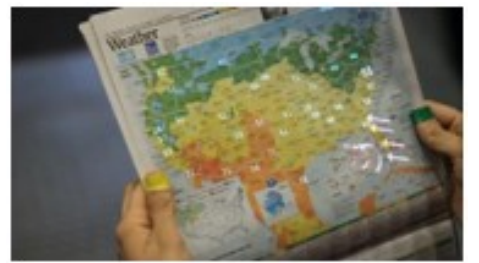

Fig 11.Weather Information

\section{ADVANTAGES}

The interface of insight is straightforward to use and it classifies digital information into physical world.

Hence, the entire world may be used as associate degree interface creating the entire world your machine [9]. it's straightforward to hold device, supports multi-touch, simply cheap, world and also the info square measure connected to every alternative, information may be accessed in globe, helps North American nation in creating right selections, it acts a laptop also as saves our time looking info on the net.

Sixth Sense uses hand gestures to interface with digital information. Info is directly displayed from digital world to physical world. It's a wearable gesture primarily based device which might flip associate degree surface an intuitive presentation. It's compact and simple to wear in our neck. This device may be utilized by anybody, even the one World Health Organization don't shrewdness to use an electronic device or console or keyboard. In today's contemporary world it'll be straightforward to catch pictures simply by victimisation fingers.

\section{DISADVANTAGES}

HARDWARE LIMITATIONS OF THE DEVICES THAT WE TEND TO PRESENTLY CARRY AROUND WITH U.S.A. FOR INSTANCE SEVERAL PHONES WON'T PERMIT THE EXTERNAL CAMERA FEED TO BE MANIPULATED IN REAL TIME.

\section{FUTURE ENHANCEMENT}

To get obviate color markers.

To incorporate camera and projector within mobile machine.

To have 3D gesture following.

To make intuition work as fifth Sense for disabled person.

\section{CONCLUSION}

SiXTH SENSE ACKNOWLEDGES THE OBJeCtS AROUND U.S., DISPLAYING INFO MECHANICALLY AND PROPERTY U.S. TO ACCESS IT IN ANY METHOD WE WANT. THE INTUITION IMAGE IMPLEMENTS MANY APPLICATIONS THAT DEMONSTRATE THE UTILITY, VIABILITY AND ADAPTABILITY OF THE SYSTEM. THE POTENTIAL OF CHANGING INTO THE LAST WORD "TRANSPARENT" COMPUTER PROGRAMME FOR ACCESSING INFO CONCERNING EVERYTHING AROUND U.S. PERMITTING U.S. TO MOVE WITH THIS INFO VIA NATURAL HAND GESTURES. 


\section{Acknowledgment}

We are very thankful to Dr. Mohan Babu GN Sir, Principal of Information Sciences Department, Dr. Manjunath BMS Institute of technology and Management, Avalahalli, Bengaluru, for his valuable comments and suggestion throughout writing of this paper. This helped us in improving the quality of this paper.

\section{References}

1. G. Eason, B. Noble, and I. N. Sneddon, "On certain integrals of Lipschitz-Hankel type involving products of Bessel functions," Phil. Trans. Roy. Soc. London, vol. A247, pp. 529-551, April 1955. (References)

2. J. Clerk Maxwell, A Treatise on Electricity and Magnetism, 3rd ed., vol. 2. Oxford: Clarendon, 1892, pp.68-73.
3. I. S. Jacobs and C. P. Bean, "Fine particles, thin films and exchange anisotropy," in Magnetism, vol. III, G. T. Rado and H. Suhl, Eds. New York: Academic, 1963, pp. 271-350.

4. K. Elissa, "Title of paper if known," unpublished.

5. R. Nicole, "Title of paper with only first word capitalized," J. Name Stand. Abbrev. in press.

6. Y. Yorozu, M. Hirano, K. Oka, and Y. Tagawa, "Electron spectroscopy studies on magneto-optical media and plastic substrate interface," IEEE Transl. J. Magn. Japan, vol. 2, pp. 740-741, August 1987 [Digests 9th Annual Conf. Magnetics Japan, p. 301, 1982].

7. M. Young, The Technical Writer's Handbook. Mill Valley, CA: University Science, 1989.

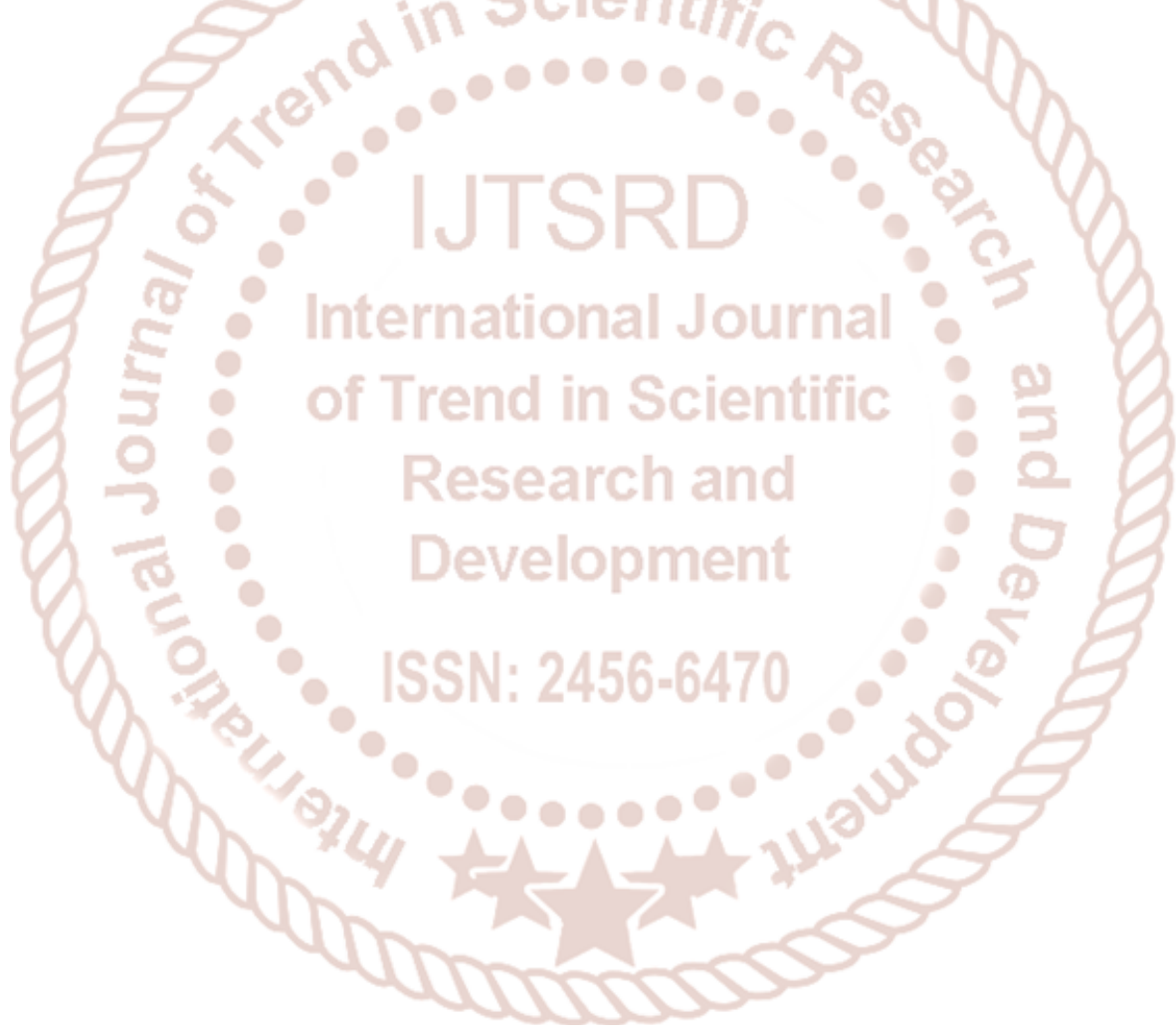

